\title{
Transcriptionally less active prodynorphin promoter alleles are associated with Temporal Lobe Epilepsy: A case-control study and meta-analysis
}

\author{
Marcelo A. Kauffman ${ }^{\mathrm{a}, \mathrm{b}, *}$, Damián Consalvo ${ }^{\mathrm{b}}$, Moron Dolores Gonzalez ${ }^{\mathrm{c}}$ and Silvia Kochen ${ }^{\mathrm{b}}$ \\ ${ }^{a}$ Consultorio de Neurogenética, Hospital Ramos Mejía, Buenos Aires, Argentina \\ ${ }^{\mathrm{b}}$ Centro de Epilepsia, División Neurología, Hospital Ramos Mejia, CEFYBO, CONICET, Buenos Aires, Argentina \\ ${ }^{\mathrm{c}}$ Residencia de Neurología, División Neurología, Hospital Ramos Mejia, Buenos Aires, Argentina
}

\begin{abstract}
We performed an association study in a population of patients with Mesial Temporal Lobe Epilepsy (TLE) with Hippocampal Sclerosis (MTEHS) together with a systematic revision of the literature to investigate the role of transcriptionally less active polymorphic alleles of Prodynorphin (PDYN) gene in this pathology. We included 102 patients with a diagnosis of MTEHS and 86 healthy controls. The positive antecedent of family history for epileptic events defined a TLE subgroup with familial predisposition for epileptic disorders. The PDYN promoter polymorphism was genotyped by means of a PCR assay. For meta-analysis, we identified case-control association studies between TLE and PDYN by searching PUBMED. The pooled OR was estimated using a fixed effects model under dominant and co-dominant heredity models. No differences in genotypic and allelic frequencies were found between cases and controls $(p=0.61)$ in our population, neither in the whole cohort nor in the analysis limited to TLE with familial predisposition $(p=0.71)$. The Meta-Analysis included 591 TLE patients and 1117 healthy controls. We found an association between L allele ( $p=0.003$; OR $=1.40$; IC $95=1.12-1.74)$ and a modestly higher risk to develop TLE in the group of patients with familial predisposition. Therefore, functional allelic variants in the PDYN promoter might modify the risk to develop TLE in subjects with familial predisposition.
\end{abstract}

Keywords: Genetics, prodynorphin, temporal lobe epilepsy, polymorphism, meta-analysis

\section{Introduction}

Traditionally, Temporal Lobe Epilepsy (TLE) has been considered to be an acquired disorder secondary to lesions. However, the observations of familial monogenic forms [1], the frequent presence of positive familial antecedents of epileptic events [2] and the identification of common variants in different genes as risk

*Corresponding author: Marcelo A. Kauffman, MD, MSc., Consultorio de Neurogenética, División Neurología, Hospital JM Ramos Mejía, Urquiza 609, 1221 Buenos Aires, Argentina. Tel./Fax: +54 114127 0280; E-mail: marcelokauffman@gmail.com. factors [3] highlight an evolving key role of genetics in TLE.

Prodynorphin (PDYN) is an opioid peptide precursor. Dynorphins, products of PDYN cleavage, are heavily expressed in the hippocampus in response to focal epileptic discharges where they might have a role in regulatory mechanisms involved in the control of neuronal hypersynchrony [4]. The PDYN promoter exhibits a functional repetitive element polymorphism that has been associated with TLE development [5]. However, this finding could have not been replicated by others raising controversy about the real role of this genetic variant in the susceptibility to develop the disease [6-8]. Persistent difficulties in obtaining robust and replicable 
results in genetic association studies are almost certain because genetic effects are small, requiring studies with many thousands of subjects to be detected [9]. Casecontrol studies are the most widely applied strategy of association studies for characterizing the genetic contribution to common diseases, although this approach is prone to lack enough sensitivity to identify putative gene variants. This difficulty can be solved, at least in part, by doing a systematic review of the literature and, therefore, performing a meta-analysis [10].

Our aims were to independently replicate the original association of PDYN promoter polymorphism with TLE and to perform a systematic review of the literature and a meta-analysis on this topic incorporating our findings in order to better estimate the role of this genetic variant in TLE. We also included an exploratory analysis in other phenotypic sub-groups.

\section{Materials and methods}

\subsection{Patients}

Between August 2003 and July 2005, we recruited one-hundred-and-two consecutive Mesial Temporal Lobe Epilepsy with Hippocampal Sclerosis (MTEHS) patients from the Epilepsy Clinic at the Neurology Division of the Ramos Mejia Hospital in Buenos Aires, Argentina. The study was reviewed and approved by the local ethics committee and a written informed consent was obtained from each patient prior to any sample recovery. All patients had a comprehensive diagnostic evaluation, including detailed seizure history and neurological examination, neuropsychological testing, optimized MRI study, and surface EEG. Long-term videoEEG monitoring was performed in a subset of patients. Data from all these patients was consistent with the diagnosis of typical MTEHS. Eighty-six healthy unrelated subjects were selected as controls. Selection of the controls was based on the lack of any positive antecedent of seizure events or other neurological or psychiatric disorder. Cases and controls were interviewed about ethnic background. We paid special attention to the nationalities of parents and grandparents of the subjects included. Control subjects were matched for age, gender, ethnic origin (nationality of parents and grandparents), and area of residence. The following clinical features were analyzed: age, sex, age at onset of epilepsy, prior history of febrile seizures, positive family history for epilepsy and febrile seizures, clinical semiology of seizures, seizure frequency, and response to pharmacological treatment.

\subsection{Genotyping}

Genomic DNA was isolated from whole blood using a Flexigene kit, as described by the manufacturer (Qiagen, Hilden, Germany). The PDYN repetitive element promoter polymorphism was genotyped by PCRethidium bromide stained agarose electrophoresis, as previously described [5]. For analysis, we considered alleles of one and two repetitions as low activity alleles (L) and alleles of three and four repetitions as high activity alleles $(\mathrm{H})$. The genotyping reactions were performed in a blinded manner to clinical features, in batches containing equal number of samples from patients and controls.

\subsection{Statistical analysis}

The Hardy-Weinberg equilibrium in the cohort of healthy controls was tested using exact test. Categorical variables were compared with the $\mathrm{X}^{2}$ test or Fisher exact test. We calculated ORs as measures of effect. A level ( $p$ value) of $<0.01$ was considered as significant, as standardized for genetic association studies. All $p$ values were two-tailed. Differences in quantitative variables were assessed with the Mann-Whitney U test, since data did not have a normal distribution. All statistical analyses were performed using Stata version 9 for Windows.

\subsection{Meta-analysis}

\subsubsection{Search strategy}

We searched MEDLINE (US National Library of Medicine) for all relevant articles published January 1966 through August 1, 2006, using the PubMed search engine. The search strategy was "Temporal Lobe Epilepsy" or "Seizure" AND "Prodynorphin" or "PDYN". There were no language or country restrictions. We included any human population-based association study, regardless of sample size, if the investigators (a) determined the association between the PDYN gene promoter repetitive element polymorphism and TLE; (b) the alleles and genotypes for this polymorphism were, respectively: L and $\mathrm{H}$; and LL, HL and $\mathrm{HH}$; (c) the outcome was TLE and there were at least two comparison groups (e.g., TLE vs. control (nonTLE) groups) and (d) there were sufficient results for extraction of data (i.e., the number of subjects with each genotype in the TLE and control groups). We also reviewed the reference lists of the retrieved articles to identify publications on the same topic. The most complete and recent results were used when there were multiple publications from the same study group. 


\subsubsection{Data extraction}

Data were extracted independently and in duplicate by two authors (M.K. and D.G.M.) using a standardized data extraction form. Data on co-variables such as publication year, nationality of subjects, gender and number of patients with positive antecedents of epileptic events were also extracted for each study. Any disagreement was adjudicated by a third author (D. C.).

The quality of the studies was assessed using a modified version of a quality assessment score developed for genetic association studies [11]. Total scores ranged from 0 (worst) to 10 (best).

\subsubsection{Statistical analysis}

Hardy-Weinberg equilibrium was assessed for each study using the exact test. We built 2 by 2 tables for each included study defining as exposed the genotypes that included the L allele and as no exposed the genotypes that did not include the L allele. As an exception, in the comparison between homozygotes for $\mathrm{L}$ alleles with heterozygotes for $\mathrm{H}$ allele, this last genotype (HL) was considered as no exposed. We imported these tables into STATA where we used the "metan $a b$ $c d$ " command [12] to calculate individuals and pooled odds ratios by means of the fixed effect inverse variance method. Thus, we performed 3 comparisons (LL vs HL; HL vs HH y LL vs HL) that defined that the dominant and co-dominant heredity models as the ones that better adjust the data. Therefore, the comparison was coalesced into LL $+\mathrm{HL}$ vs $\mathrm{HH}$ (dominant model) and L vs H (co-dominant model) contrasts. Publication bias was assessed using Egger's and Begg-Matzumdar tests. We assessed heterogeneity by using Q statistics.

\section{Results}

\subsection{Our population}

The distribution of genotypes and allele frequencies of the PDYN promoter polymorphism in patients and controls is summarized in Table 1. The LL, HL and $\mathrm{HH}$ genotypes were observed in 9 (8.8\%), 40 (39.2\%) and $53(52 \%)$ patients and in $8(9.3 \%), 37(43 \%)$ and $41(47.7 \%)$ healthy controls, respectively. Thus, the allelic and genotypic frequencies were not different ( $p=$ 0.61 ). The observed genotype distributions in the cohort of controls and the respective theoretical distributions inferred from the observed allele frequencies were not significantly different (Hardy-Weinberg equilibrium). We did not examine other genetic variants.
The analysis limited to the sub-group of patients with familial predisposition to suffer from epileptic disorders, i.e., those with a positive familial history of seizures, did not show that this polymorphism was associated with TLE $(p=0.71)$ (see Table 1$)$.

In the exploratory analysis, we investigated the effect of the PDYN polymorphism on parameters of clinical characteristics of the syndrome. The proportion of patients experiencing frequent nocturnal seizures was significantly higher in the HH genotype group compared to the $\mathrm{L}$ carriers genotypes group ( $p=0.0001)$. In contrast, we did not find any association between these allelic variants and any other of the characteristics investigated (see Table 2).

\subsection{Meta-analysis}

Nineteen studies were identified by our search strategy. Fifteen of these studies were not eligible, leaving 4 studies for inclusion in this analysis. From these studies, a total of 489 cases and 1031 control subjects have been analyzed for disease association between TLE and PDYN genetic variation. As we included our own study cohorts, the overall total added up to 591 TLE cases and 1117 control subjects. All studies were carried out in Caucasians. The quality of studies ranged from 5 to 10 , out of a possible score of 10 . In all studies, investigators used the same DNA genotyping method.

In order to produce a synthesis of the data from multiple studies, we undertook a meta-analysis of the association between PDYN promoter polymorphism and TLE in 5 studies including our own study (see Table 3 ). We performed two analyses, first we considered the whole population of TLE patients and then we restricted our comparison to data from TLE patients with familial predisposition to epileptic disorders (see results in our population). There was no evidence of heterogeneity between the studies included ( $p=0.359$ for TLE and $p=0.221$ for TLE with familial predisposition). We did not find any association between TLE and PDYN promoter polymorphism $(p=0.69$; OR $=$ 1.04 ; CI $95=0.84-1.29$ and $p=0.54$; OR $=1.05$; CI $95=0.89-1.24$ for dominant and co-dominant models, respectively). By contrast, we found that TLE was associated with PDYN promoter gene variants in subjects with a familial predisposition to suffer from epileptic disorders $(p=0.01$; OR $=1.44$; CI $95=1.06-1.95$ and $p=0.003$; OR $=1.40 ;$ CI $95=1.12-1.74$ for dominant and co-dominant models, respectively) (see Fig. 1). There was not evidence of publication bias in both analysis (Begg's test: $p=0.80$ and Egger's test: $p=0.49$, Begg's test: $p=1$ and Egger's test: $p=$ 0.82 , respectively). 
Table 1

Genotypic frequencies in our population

\begin{tabular}{llll}
\hline Genotypes & MTEHS & FMTEHS & Controls \\
& $\mathrm{N}=102(\%)$ & $\mathrm{N}=18(\%)$ & $\mathrm{N}=86(\%)$ \\
\hline $\mathrm{LL}$ & $9(8.8)$ & $1(5.5)$ & $8(9.3)$ \\
$\mathrm{HL}$ & $40(39.2)$ & $8(44.5)$ & $37(43)$ \\
$\mathrm{HH}$ & $53(52)$ & $9(50)$ & $41(47.7)$ \\
OR $(95 \%$ IC) & $0.89(0.57-1.38)^{*}$ & $1.15(0.52-2.53)^{*}$ & \\
$P$ Value & $0.61^{* *}$ & $0.71^{* *}$ & \\
\hline
\end{tabular}

MTEHS Mesial Temporal Lobe Epilepsy with Hippocampal Sclerosis. FMTEHS Familial Mesial Temporal Lobe Epilepsy with Hippocampal Sclerosis.

FH Positive Family History for epileptic events.

* OR Crude Odds Ratio.

${ }^{* *} \mathrm{X}^{2}$ Test.

Table 2

Variables studied in patients with MTEHS

\begin{tabular}{llll}
\hline Variables & LL + HL & HH & $p$ Value \\
& $\mathrm{N}=59$ & $\mathrm{~N}=52$ & \\
\hline Men, n & 25 & 29 & $\mathrm{NS}$ \\
Women, n & 24 & 24 & $\mathrm{NS}$ \\
Age of Onset mean in years & 15.02 & 16.69 & $\mathrm{NS}$ \\
Antecedent FC, n (\%) & $13(48.3)$ & $18(34.6)$ & $\mathrm{NS}$ \\
Positive FH for EE, n (\%) & $9(15.2)$ & $9(17.3)$ & $\mathrm{NS}$ \\
Status epilepticus, n (\%) & $8(13.55)$ & $14(26.9)$ & $\mathrm{NS}$ \\
Clustering of seizures, n (\%) & $8(13.55)$ & $9(17.3)$ & $\mathrm{NS}$ \\
Antecedent NS, n $(\%)$ & $16(27.1)$ & $36(69.23)$ & $0.0001^{*}$ \\
Seizures/month at investigation & $1-2$ & $3-4$ & $\mathrm{NS}$ \\
Refractory, n $(\%)$ & $28(47.4)$ & $28(53.8)$ & $\mathrm{NS}$ \\
\hline
\end{tabular}

${ }^{*} \mathrm{X}^{2}$ Test.

$\mathrm{FC}=$ Febrile Convulsions.

$\mathrm{EE}=$ Epileptic Events.

$\mathrm{NS}=$ Nocturnal Seizures

Table 3

Studies included in meta-analysis

\begin{tabular}{|c|c|c|c|c|c|c|c|c|c|c|c|c|}
\hline Author/Country/Year & TLEHH & TLEHL & TLELL & Totcases & FTLEHH & FTLEHL & FTLELL & ContHH & ContHL & ContLL & Totcont & Quality* \\
\hline Stögmann/Austria/2002 & 60 & 69 & 16 & 145 & 10 & 23 & 10 & 96 & 88 & 18 & 202 & 8 \\
\hline Tilgen/Germany/2003 & 90 & 78 & 14 & 182 & 22 & 21 & 3 & 99 & 84 & 22 & 205 & 5 \\
\hline Gambardella/Italy/2003 & 66 & 40 & 9 & 115 & 28 & 25 & 7 & 138 & 105 & 16 & 259 & 8 \\
\hline Cavalleri/UK/2005 & 17 & 22 & 8 & 47 & 17 & 22 & 8 & 175 & 160 & 30 & 365 & 8 \\
\hline Kauffman/Argentina/2006 & 53 & 40 & 9 & 102 & 9 & 8 & 1 & 41 & 37 & 8 & 86 & 10 \\
\hline
\end{tabular}

TLEHH: Temporal Lobe Epilepsy genotype HH; TLEHL: Temporal Lobe Epilepsy genotype HL; TLELL: Temporal Lobe Epilepsy genotype LL; Totcases: cases included; FTLEHH: Familial Temporal Lobe Epilepsy genotype HH; FTLEHL: Familial Temporal Lobe Epilepsy genotype HL; FTLELL: Familial Temporal Lobe Epilepsy genotype LL; ContHH: Controls with genotype HH; ContHL: Controls with genotype HL; ContLL: Controls with genotype LL; Totcont: Controls included.

* Quality score as desribed in methods.

\section{Discussion}

We demonstrate a modest association between transcriptionally less active alleles of PDYN and TLE in subjects familiarly predisposed. This finding may contrasts previous studies, including the analysis in our own population, but it is similar to the results of the first research on this topic performed by Stogmann et al. [5]. For instance, Tilgen, Cavalleri and Gambardella inves- tigated the role of PDYN promoter repetitive element polymorphism in TLE where they did not found an overall association in different European patients [68]. However, this apparent contradiction is very often found in the field of genetics of complex disorders. Discrepant findings may be due to multiple causes such as differences in the populations analyzed, difficulties with the phenotype definition or designs with low power to detect genetic effects that necessarily are 


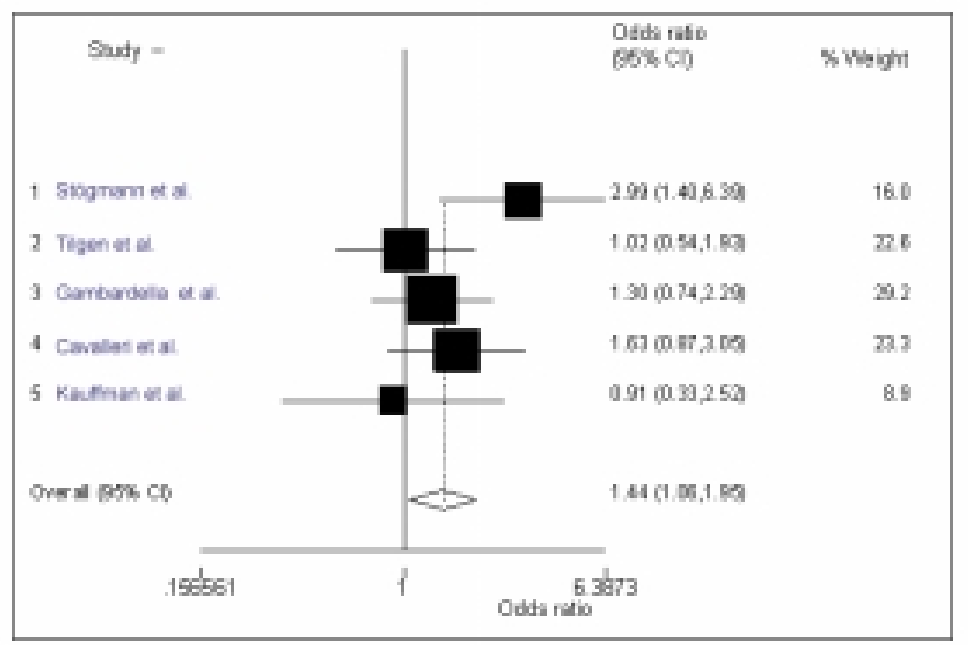

Fig. 1. Forest Plot Familial Temporal Lobe Epilepsy under dominant heredity model.

small [13]. This difficulty could be solved, at least in part, by making a systematic review of the literature and, thus, performing a meta-analysis. Nevertheless, the different results obtained in the populations investigated could depend on epistatic interactions between PDYN and other putative susceptibility genes with particular geographic prevalence. Moreover, the observed association was selectively present in genetically predisposed individuals indicating that a multiplicative interaction with other susceptibility genes could be required to reach the threshold for TLE.

Of course the results of any meta-analysis must always be viewed with caution; it is thought that all studies to date investigating the PDYN promoter gene variant in TLE were included in this work. However, unpublished negative work may result in selection bias, but this is unlikely as even the so called negative genetic associations still attract interest and we attempted to limit the impact of this problem as described in the Method section. Nevertheless, it is more likely that ongoing studies have not been included, but the combined power of this meta-analysis is probably greater than any single continuing study. Since a sensitivity analysis (data not shown) that excluded the data from Stogmann's work resulted in a lack of significance for the association, a word of caution has to be paid to the robustness of our findings. However, similar to many candidate genes influencing complex disorders susceptibility, the PDYN variant genetic effect is modest and requires thorough evaluation in other independent populations.

In contrast to other polymorphisms associated with TLE, the promoter variant in PDYN seems to be func- tionally active [14]. Moreover, independent lines of phylogenetic and population genetic evidence support a history of selective sweeps driving the evolution of the human PDYN promoter that underscore that this polymorphism has been and still is functionally relevant in humans [15]. Alleles with a lower number of repetitions are transcriptionally less active than alleles with a higher number. Moreover, PDYN promoter has an AP-1 binding site. Taking into account that AP-1 might be central in transcriptional cascades that follow an induced seizure in epilepsy experimental models [16] and that dynorphins have an important role in neuronal homeostasis regulating the opening of $\mathrm{K}+$ channels [4], it can be suggested that functional variants in PDYN could be plausibly involved in TLE epileptogenesis. Furthermore, dynorphins are extensively expressed in dentate gyrus' granular cells [17]. Alternatively, PDYN promoter variants might be not only relevant for temporal lobe epilepsy but also for all forms of epilepsies, as Cavalleri et al. suggested [7]. Since a higher extent of neuronal synchrony is observed during slow sleep [18] and a higher level of PDYN transcription might be compensatory of this hypersynchrony, the observed association between $\mathrm{H}$ alleles and nocturnal seizures is intriguing. Given that this finding is part of our exploratory analysis, it must be only considered as hypothesis generating and it deserves an independent replication.

In summary, to the best of our knowledge, the available evidence to date indicates that subjects with a familial predisposition to epileptic disorders carrying $\mathrm{L}$ alleles of PDYN gene promoter have, at least, a $40 \%$ 
greater risk to suffer from TLE than those homozygous for the $\mathrm{H}$ alleles.

\section{Acknowledgements}

We would like to thank the patients and their families for their kindest cooperation with this research. MA Kauffman, MD was supported by a PhD. scholarship from CONICET (Consejo Nacional de Investigaciones Científicas y Técnicas, Argentina).

\section{References}

[1] C.V. Maurer-Morelli, R. Secolin, R.B. Marchesini, N.F. Santos, E. Kobayashi, F. Cendes and I. Lopes-Cendes, THE SCN2A gene is not a likely candidate for familial mesial temporal lobe epilepsy, Epilepsy Res 71 (2006), 233-236.

[2] R.S. Briellmann, Y. Torn-Broers, B.E. Busuttil, B.J. Major, R.M. Kalnins, M. Olsen, G.D. Jackson, A.G. Frauman and S.F. Berkovic, APOE epsilon4 genotype is associated with an earlier onset of chronic temporal lobe epilepsy, Neurology $\mathbf{5 5}$ (2000), 435-437.

[3] N.C. Tan, J.C. Mulley and S.F. Berkovic, Genetic association studies in epilepsy: "the truth is out there", Epilepsia $\mathbf{4 5}$ (2004), 1429-1442.

[4] M.V. Solbrig, R. Adrian, D.Y. Chang and G.C. Perng, Viral risk factor for seizures: Pathobiology of dynorphin in herpes simplex viral (HSV-1) seizures in an animal model, Neurobiol Dis (2006).

[5] E. Stogmann, A. Zimprich, C. Baumgartner, S. AullWatschinger, V. Hollt and F. Zimprich, A functional polymorphism in the prodynorphin gene promotor is associated with temporal lobe epilepsy, Ann Neurol 51 (2002), 260-263.

[6] A. Gambardella, I. Manna, A. Labate, R. Chifari, P. Serra, A. La Russa, E. LePiane, R. Cittadella, V. Andreoli, F. Sasanelli, M. Zappia, U. Aguglia and A. Quattrone, Prodynorphin gene promoter polymorphism and temporal lobe epilepsy, Epilepsia 44 (2003), 1255-1256.
[7] G.L. Cavalleri, J.M. Lynch, C. Depondt, M.W. Burley, N.W. Wood, S.M. Sisodiya and D.B. Goldstein, Failure to replicate previously reported genetic associations with sporadic temporal lobe epilepsy: where to from here?, Brain 128 (2005), 1832-1840.

[8] N. Tilgen, J. Rebstock, S. Horvath, P. Propping, C.E. Elger and A. Heils, Prodynorphin gene promoter polymorphism and temporal lobe epilepsy, Ann Neurol 53 (2003), 280-281; author reply $1-2$.

[9] N.C. Tan, J.C. Mulley and I.E. Scheffer, Genetic dissection of the common epilepsies, Curr Opin Neurol 19 (2006), 157163.

[10] M.R. Munafo, Candidate gene studies in the 21st century: meta-analysis, mediation, moderation, Genes Brain Behav 5(suppl 1) (2006), 3-8.

[11] A. Thakkinstian, C. D'Este, J. Eisman, T. Nguyen and J. Attia, Meta-analysis of molecular association studies: vitamin D receptor gene polymorphisms and BMD as a case study, $J$ Bone Miner Res 19 (2004), 419-428.

[12] M. Bradburn, metan-An alternative meta-analysis command, in 2006.

[13] K.T. Zondervan and L.R. Cardon, The complex interplay among factors that influence allelic association, Nat Rev Genet 5 (2004), 89-100.

[14] A. Zimprich, J. Kraus, M. Woltje, P. Mayer, E. Rauch and V. Hollt, An allelic variation in the human prodynorphin gene promoter alters stimulus-induced expression, J Neurochem $\mathbf{7 4}$ (2000), 472-477.

[15] M.V. Rockman, M.W. Hahn, N. Soranzo, F. Zimprich, D.B. Goldstein and G.A. Wray, Ancient and recent positive selection transformed opioid cis-regulation in humans, PLoS Biol 3 (2005), e387.

[16] K. Ogita, T. Kitayama, H. Okuda and Y. Yoneda, Effects of glutathione depletion by 2-cyclohexen-1-one on excitatory amino acids-induced enhancement of activator protein-1 DNA binding in murine hippocampus, J Neurochem 76 (2001), 19051915.

[17] F. Merg, D. Filliol, I. Usynin, I. Bazov, N. Bark, Y.L. Hurd, T. Yakovleva, B.L. Kieffer and G. Bakalkin, Big dynorphin as a putative endogenous ligand for the kappa-opioid receptor, $J$ Neurochem 97 (2006), 292-301.

[18] B.A. Malow, Sleep and epilepsy, Neurol Clin 23 (2005), $1127-$ 1147. 


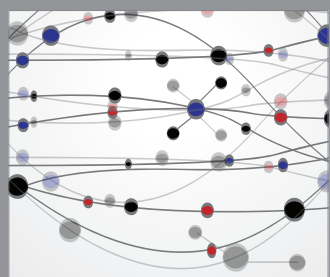

The Scientific World Journal
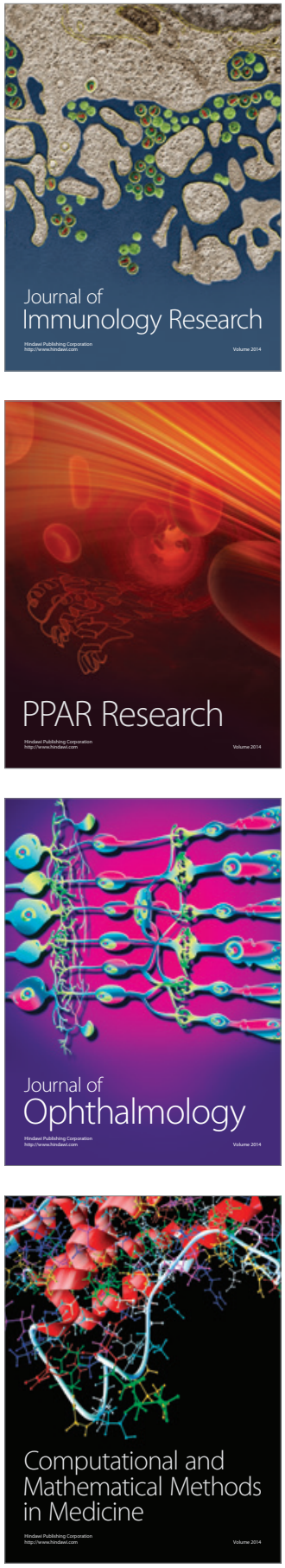

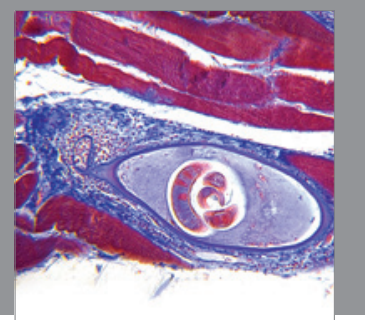

Gastroenterology

Research and Practice
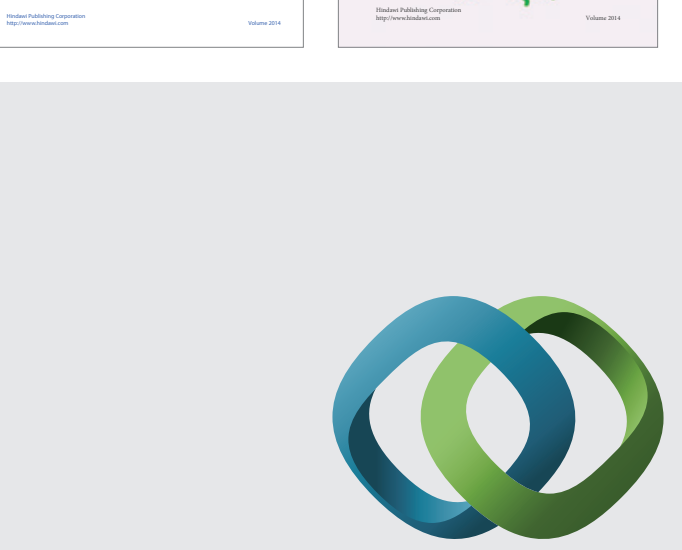

\section{Hindawi}

Submit your manuscripts at

http://www.hindawi.com
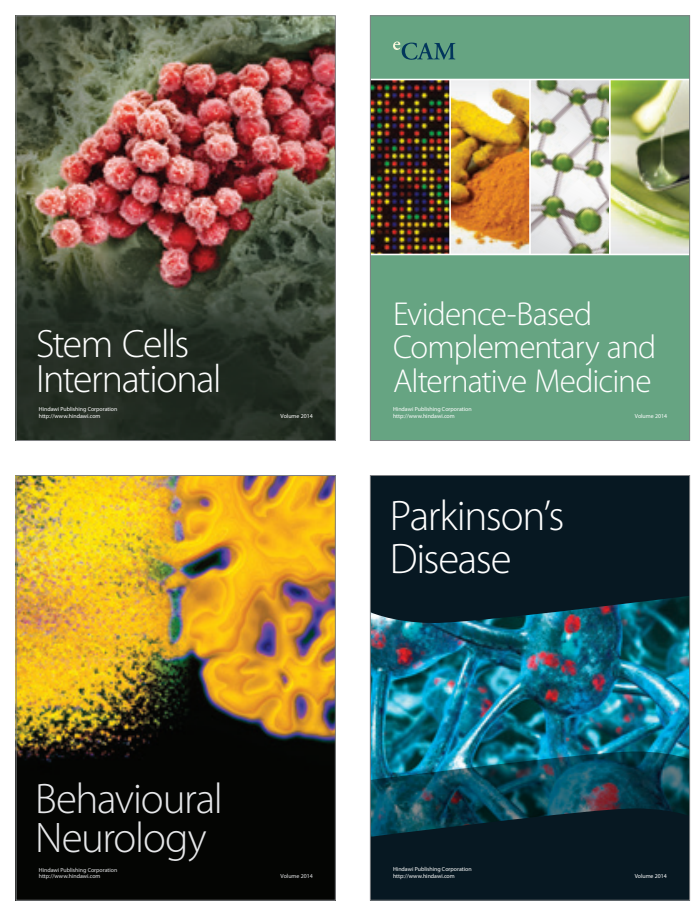

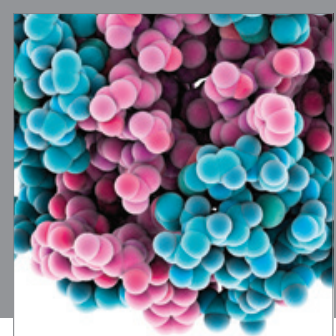

Journal of
Diabetes Research

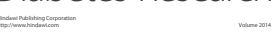

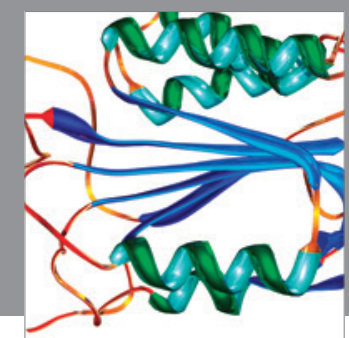

Disease Markers
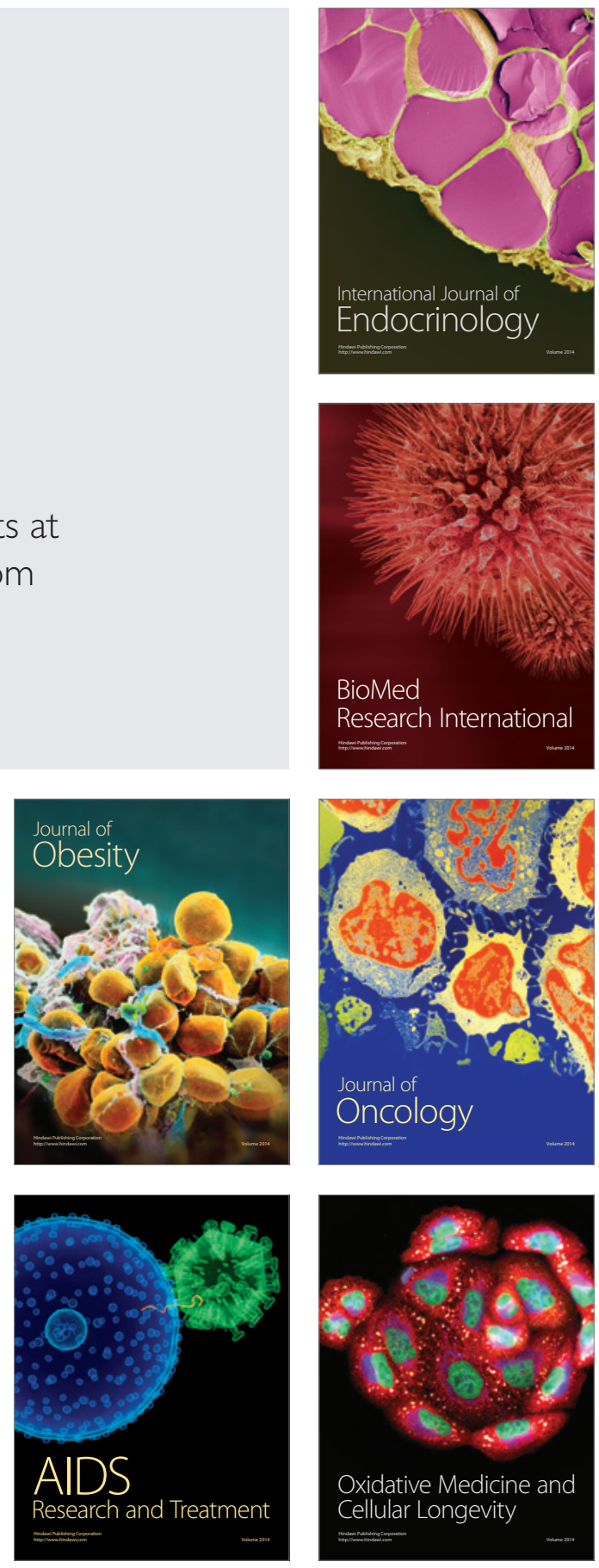